\title{
ASPECTS OF MODEL-BASED DECOMPOSITIONS IN RADAR POLARIMETRY
}

\author{
A.P. Doulgeris and T. Eltoft \\ The Department of Physics and Technology \\ UiT- The Arctic University of Norway \\ Troms $\varnothing$, Norway
}

\begin{abstract}
In this paper, we further analyse the problem that polarimetric target decomposition methods in general have more physical parameters than equations, making the decomposition underdetermined and hence have no unique solution. The common approach to get around this problem is to make certain assumptions, thus fixing one or more parameters, allowing the other free parameters to be solved from the set of expressions. We recently showed how to obtain additional information from fourth-order statistics to find a unique solution to model-based polarimetric decompositions ([1]). We previously showed a fourth-order unique solution that was valid only for Gaussian data, and indicated that non-Gaussian data led to an over-estimation in many of the parameters. This work describes our new method to obtain a generic textured data solution through an optimisation approach and presents preliminary results for a sea ice specific model.
\end{abstract}

Index Terms - Polarimetric Decomposition, Product Model, Scattering mechanisms, Statistics, Optimisation, Sea ice

\section{INTRODUCTION}

The backscatter of SAR signals from Arctic sea ice, and many other rough surfaces, is thought to be caused by a combination of several scattering mechanisms, in general categorised as surface scattering, volume scattering, and double bounce scattering. SAR polarimetry has the capability of decomposing the signals into components representing these specific mechanisms. One class of polarimetric decomposition theorems is denoted model-based decompositions, in which, a physical model is connected to the various scattering mechanisms. The Freeman-Durden three-component model [2] is a well-known example of a model-based decomposition algorithm, where a specific parameterised model is associated with the covariance matrix of each of the above-mentioned components. There is experimental evidence that electromagnetic backscatter from sea ice in some cases has a high degree of depolarisation. Depolarisation is in some decomposition models mostly attributed to volume scattering. However, for sea ice backscattering it is anticipated that depolarisation can also be caused by surface effects related to rough and highly deformed ice, as well as by volume type scattering from brine inclusions and inhomogeneities inside the ice column.

Previous analysis of polarimetric decompositions of sea ice scenes using existing algorithms show that the power of the double bounce component often is an order of magnitude below the contribution from surface and volume scattering, and that high double bounce is restricted to isolated features [3]. Hence, we will in this study explore model-based polarimetric decomposition using a model which consists of only two terms; a surface scattering component and a volume scattering component. The surface component is to be described by the extended Bragg (X-Bragg) model [4], which can also predict surface based depolarisation. The volume component is modelled as an azimuth symmetric scattering mechanism, consisting of randomly oriented scatterers, geometrically categorised with a single shape parameter. In total, the model for the polarimetric coherence matrix has 6 unknowns, but only 5 independent equations to solve for the parameters. Hence, the system is underdetermined like many decomposition models.

In [1] we explored how higher-order statistical moments can add information, and be used to obtain a determined set of equations. We have found that considering statistical component mixtures leads to the same decomposition equations for the second-order covariance or coherency matrices, and importantly gives extra unique equations for the fourth-order marginals that can be combined to find a unique solution. In addition, the fourth-order expressions are quite generic, add only one additional texture parameter, and are found in terms of the second-order elements that fully define the Gaussian speckle scattering distributions. We have previously only shown a solution for purely Gaussian scattering coefficients and demonstrated that radar texture would lead to over-estimates of many of the parameters. In addition the estimation did not consider any random variation due the speckle, and assumed that the covariance estimate was perfect. We attempt to remedy these weaknesses here.

We will recap the theoretical aspects of our methodology, detail the new optimisation approach, and show results of preliminary studies based on simulated and real data. 


\section{BASIC METHOD}

Traditional Decompositions model the covariance or coherency matrix as a mixture of contributions

$$
\mathbf{C}=\sum_{j=1}^{N_{c}} P_{j} \tilde{\mathbf{C}}_{j} \quad \text { or } \quad \mathbf{T}=\sum_{j=1}^{N_{c}} P_{j} \tilde{\mathbf{T}}_{j}
$$

where $P_{j}$ represents the power in each component and $\tilde{\mathbf{C}}_{j}$ or $\tilde{\mathbf{T}}_{j}$ are SPAN normalised covariance or coherency matrices for each scattering model. For example, the Freeman-Durden 3-component decomposition [2] has single-bounce, doublebounce and volume components. Under such modelling the $P_{j}$ s are independent variables in the solution.

It is equivalent to convert to power fractions, such that $f_{j}=P_{j} /$ SPAN or $P_{j}=f_{j}$ SPAN, and there are still $N_{c}$ independent variables, SPAN plus $\left(N_{c}-1\right)$ fractions due to the constraint $\sum_{j=1}^{N_{c}} f_{j}=1$.

Thinking about decompositions as mixing fractions leads to a statistical interpretation as a probabilistic mixture with the implication that we may carry this mixture modelling down to the scattering vector level (s or $\mathbf{k}$ ) as, for example,

$$
p_{k}(\mathbf{k})=\sum_{j=1}^{N_{c}} f_{j} p_{j}(\mathbf{k})
$$

Furthermore, we consider the scalar product model for texture, such that

$$
\mathbf{k}=\sqrt{\tau_{j}} \mathbf{X} ; \mathbf{X} \sim \mathcal{N}_{d}^{c}\left(0, \mathbf{T}_{\mathbf{j}}\right) \text { and } E\left\{\tau_{j}\right\}=1 .
$$

Consequently, the second-order statistics are exactly the decomposition as before, since $E\left\{\tau_{j}\right\}=1$,

$$
\mathbf{T}=\sum_{j=1}^{N_{c}} f_{j} \operatorname{SPAN} \tilde{\mathbf{T}}_{j}
$$

and it follows that the fourth-order statistic in each channel is

$$
E\left\{\left|\mathbf{k}_{i}\right|^{4}\right\}=\sum_{j=1}^{N_{c}} f_{j} 2 E\left\{\tau_{j}^{2}\right\} \operatorname{SPAN}^{2} \tilde{T}_{i i, j}^{2}
$$

where $\tilde{T}_{i i, j}$ is the $i^{\text {th }}$ diagonal elements of the $j^{\text {th }}$ model matrix, and for the moment we take all $\mathrm{E}\left\{\tau_{j}^{2}\right\} \mathrm{s}$ as equal.

These are unique equations and valid for any scattering component models. Note that if we assume no texture, i.e., Gaussian vectors, then $E\left\{\tau_{j}^{2}\right\}=1$.

\section{AN EXAMPLE DECOMPOSITION}

We shall explore the two component X-Bragg model for sea ice that we have introduced before [3], which was previously used for soil moisture applications in [4]. The second-order coherency model has six parameters $\left(f_{s}\right.$, SPAN, $\delta$, and $\rho$ real, plus $\beta$ complex), but only five equations ( 3 real diagonals, plus one complex off-diagonal). The physical parameters have been described previously in [4, 1]. We extend these equations with our fourth-order expressions and the full set of equations for the decomposition are listed thus:

$$
\begin{aligned}
& \text { The second-order expressions } \\
& <T_{11}>=f_{s} \operatorname{SPAN} \frac{1}{\left(1+|\beta|^{2}\right)}+\left(1-f_{s}\right) \operatorname{SPAN} \frac{(1+\rho)}{(3-\rho)} \\
& <T_{22}>=f_{s} \operatorname{SPAN} \frac{|\beta|^{2}(1+\operatorname{sinc}(4 \delta))}{2\left(1+|\beta|^{2}\right)}+\left(1-f_{s}\right) \operatorname{SPAN} \frac{(1-\rho)}{(3-\rho)} \\
& <T_{33}>=f_{s} \operatorname{SPAN} \frac{|\beta|^{2}(1-\operatorname{sinc}(4 \delta))}{2\left(1+|\beta|^{2}\right)}+\left(1-f_{s}\right) \operatorname{SPAN} \frac{(1-\rho)}{(3-\rho)} \\
& <T_{12}>=f_{s} \operatorname{SPAN} \frac{\beta^{*}(\operatorname{sinc}(2 \delta))}{\left(1+|\beta|^{2}\right)} \\
& \quad \text { The fourth-order expressions } \\
& <k_{1}^{(4)}>=2 E\left\{\tau^{2}\right\} \operatorname{SPAN}^{2}\left(f_{s}\left[\frac{1}{\left(1+|\beta|^{2}\right)}\right]^{2}+\left(1-f_{s}\right)\left[\frac{(1+\rho)}{(3-\rho)}\right]^{2}\right) \\
& <k_{2}^{(4)}>=2 E\left\{\tau^{2}\right\} \operatorname{SPAN}^{2}\left(f_{s}\left[\frac{|\beta|^{2}(1+\operatorname{sinc}(4 \delta))}{2\left(1+|\beta|^{2}\right)}\right]^{2}+\left(1-f_{s}\right)\left[\frac{(1-\rho)}{(3-\rho)}\right]^{2}\right) \\
& <k_{3}^{(4)}>=2 E\left\{\tau^{2}\right\} \operatorname{SPAN}^{2}\left(f_{s}\left[\frac{|\beta|^{2}(1-\operatorname{sinc}(4 \delta))}{2\left(1+|\beta|^{2}\right)}\right]^{2}+\left(1-f_{s}\right)\left[\frac{(1-\rho)}{(3-\rho)}\right]^{2}\right)
\end{aligned}
$$

If we take the simple case with Gaussian component scattering vectors and assume that each multi-look coherency matrix estimate has no speckle variation, then the equations can be solved analytically and are very fast to evaluate. To demonstrate this example we choose a large number of looks to avoid too much variation, and we expect that the analytical solution will possibly produce the occasional invalid solution.

\section{OPTIMISATION APPROACH}

The more general case has unknown radar texture, i.e., nonunity $\mathrm{E}\left\{\tau^{2}\right\}$, and should account for some speckle variation. We suggest that it may be solved with a non-linear error function minimisation routine.

Our optimisation approach is to empirically build a cost function based on the total squared error from each of the relevant coherency matrix elements and fourth-order moments, essentially an error for each equation. Since the equations are essentially matching the estimated values at the pixel with the parametric expressions for each element, then a simple error is the difference between observed element and the model values. This gives four error terms for the coherency matrix equations three for the fourth-order expressions. Since the fourth-order expressions need a value for the unknown $\mathrm{E}\left\{\tau^{2}\right\}$, we could include it as an additional optimisation parameter, or use the three ratios of fourth-order terms such that the texture parameter cancels away. We have implemented the latter approach here for simplicity, although the texture estimate itself could also be interesting to interpret.

Finally, the different elements and orders may have hugely different scales and may lead to the largest element's 

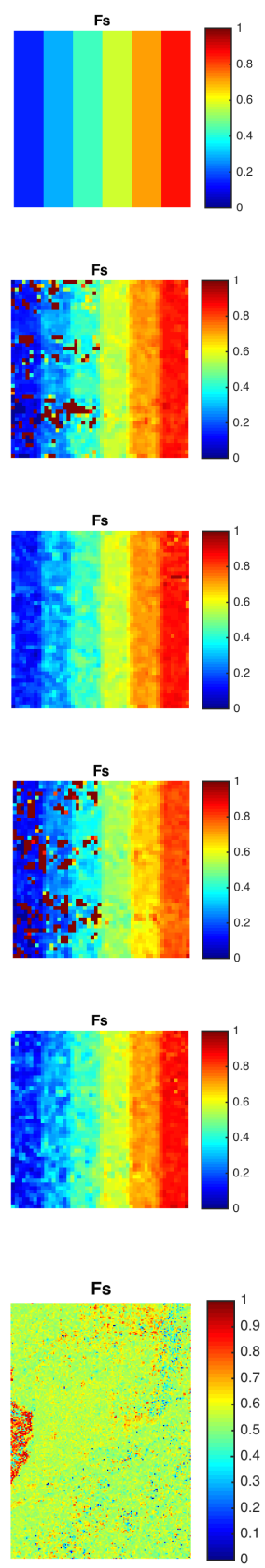
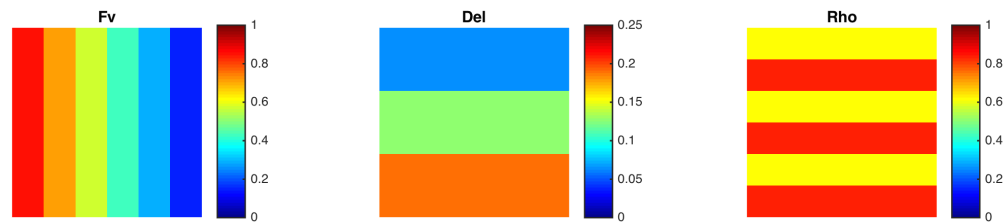

(a) Ground Truth images (fixed scales, colour reference)
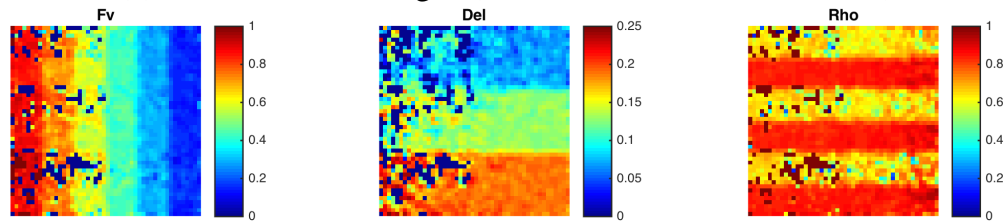

(b) Gaussian data, Gaussian algebraic solution
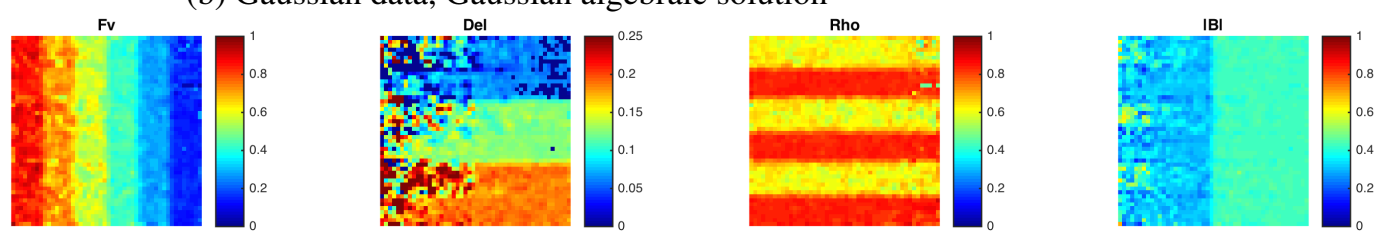

(c) Gaussian data, non-Gaussian optimised solution
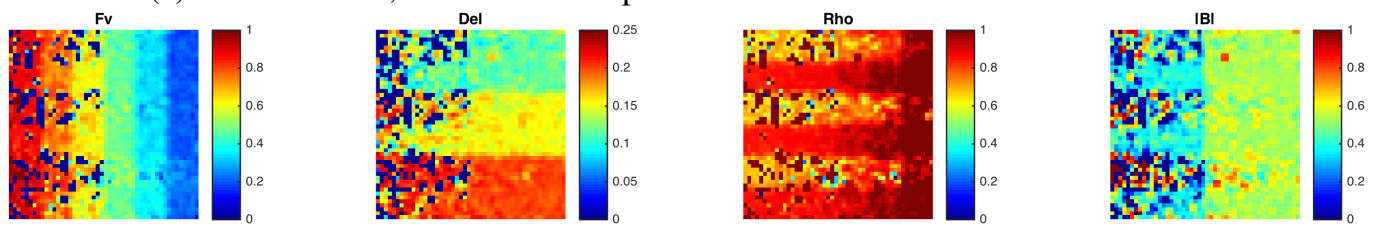

(d) Non-Gaussian data, inadequate Gaussian algebraic solution
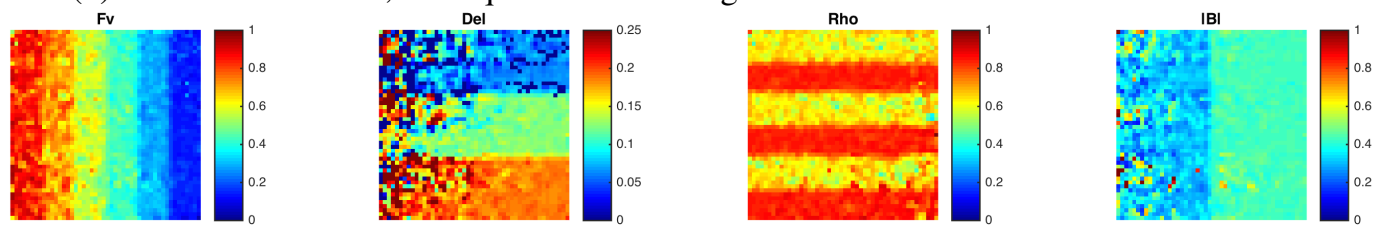

(e) Non-Gaussian data, general non-Gaussian optimised solution
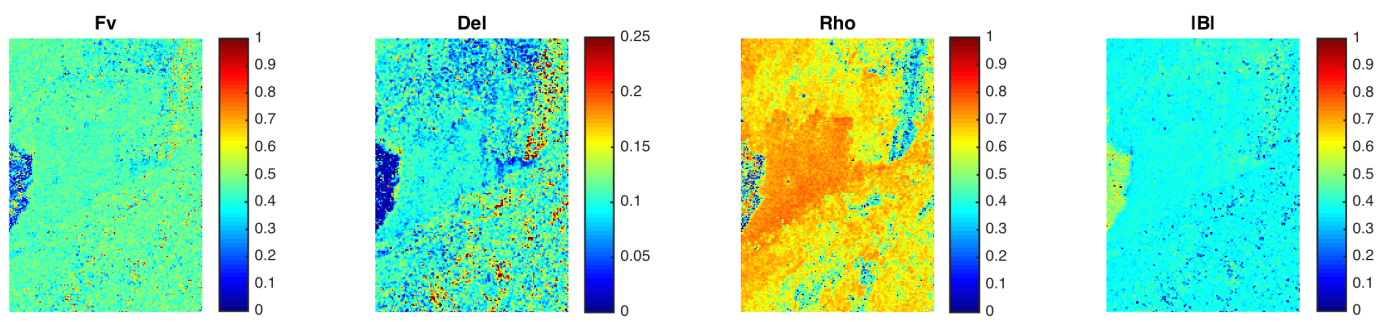

(f) Real sea ice image, Radarsat-2, general non-Gaussian optimised solution

Fig. 1. Preliminary results for the simulated test-pattern under different test conditions. The colour scale is fixed for all plots to give an immediate visual validation, and the columns represent different physical parameters. The sub-captions list the generated data type and the solution method. There are two plots with Gaussian generated data, and two with non-Gaussian data. We can see that the new optimisation approach improves the robustness through the speckle variation, and achieves the correct solution for the non-Gaussian case. The final plot is for a real sea ice scene and generally shows the expected behaviour. 
error dominating the total error function and would lead to some equations being essentially ignored. Hence, we have tried to normalise the error terms such that each error is approximately equal, and hence all the equation should have a balanced influence on the solution. We currently use an empirical approach, where the element sample variance is calculated from a local window, but we hope in the future to determine theoretical variance estimates under the model assumptions. In addition we have applied some simple square-root transforms to make the error terms more symmetric and presumably more robust, and these were verified experimentally from the local histograms. This approach seems to achieve good results the simulated data verification.

\section{PRELIMINARY RESULTS}

We use our previous coherency matrix blocked test-pattern, with different parameter values within the valid range of parameter values for sea ice and ocean properties. We generated data for each block of $200 \times 200$ pixels for this simulated testpattern by a 'hard' probabilistic mixture model for the scattering vectors under the mixture model in equation (1). We then used a large number of looks, for example $50 \times 50$, to suppress the speckle variation and to get the right mixtures, on average, for the multi-look coherency matrix image, under our modelling assumptions. The fourth-order moments were calculated from the SLC scattering vectors for each dimension within each multi-look window. Finally, we use the various algorithms to try to recover the parameters and compare to the original generated values.

The results for this test-pattern are shown in Fig. 1 (a) to (e). The first row (a) shows five of the generated parameters $\left(f_{s}, f_{v}, \delta, \rho\right.$ and $\left.|\beta|\right)$, and can be visualised as the colour reference for the quality of the results since we keep all the colour scales fixed. Row (b) shows the previous results for Gaussian data using the fourth-order equations with the Gaussian analytical solution. We see the occasional random solution, which we expect is due to not accounting for the speckle induced error in the solution. Row (c) clearly demonstrates that new optimisation approach produces smoother results than the analytical solution. So far, all the generated data was Gaussian, so we next generated non-Gaussian components, with an K-distribution with moderate texture $(\alpha=10)$. Row (d) shows that non-Gaussian data produces incorrect estimates for the parameters when using the Gaussian-only analytical solution, while row (e) shows the new general result for non-Gaussain data with the non-Gaussian (textured) model via the new numerical optimisation approach. This recovers the original colours (and hence parameters) and may be used for general real PolSAR scenes.

The final experiment in Fig. 1.(f), is with real sea ice data from a Radarsat-2 scene of the Fram strait from September 2011. The scene depicts some open water, some smooth level ice, some rough deformed ice areas, and some ridges. In- terpreting the five physical parameter images, we can clearly see that many known physical relations are correctly modelled, such as water dominated by surface scattering, low $\delta$ and higher $|B|$, although the true values are unknown for this scene and cannot be validated.

\section{CONCLUSIONS}

This work presents a new optimisation approach to solve the decomposition parameters through the second-order and fourth-order expressions. This work extends the previous work that showed that the fourth-order terms determines a unique solution and finds a generic non-Gaussian, or textured, data solution via cost function optimisation. The optimisation approach solves two limitations: it now partially accounts for speckle variation; and it produces results for the non-Gaussian case that we could not solve analytically. This is only the preliminary results, but it already looks promising.

The results clearly demonstrate that the minimisation reduces some of the invalid solutions due to the speckle variation, and that the over-estimation is removed when analysing non-Gaussian, textured, data. The modelling appears to give realistic results for a real sea ice data-set, but has not yet been validated to the actual dielectric parameters, or correlated to the helicopter-borne surface roughness measurements.

The derived high-order statistical expressions are found to be independent of the actual physical model, and relate back to the second-order covariance or coherency matrices only. Hence, we believe that this approach should add value to all underdetermined decomposition schemes and this shall be tested in the future.

\section{REFERENCES}

[1] A.P. Doulgeris and T. Eltoft, "Can higher-order statistics add information in model-based polarimetric decompositions?," in Proc. POLinSAR 2015, Frascati, Italy, 26-30 January 2015, p. 6.

[2] A. Freeman and S. L. Durden, "A Three-Component Scattering model for Polarimetric SAR Data," IEEE Transactions on Geoscience and Remote Sensing, vol. 36, 3, pp. 963-973, May 1998.

[3] A.P. Doulgeris Eltoft, T and J. Grahn, "Model-based polarimetric decomposition of arctic sea ice," in Proc. EUSAR 2014 10th European Conference on Synthetic Aperture Radar, June 2014, Berlin, Germany, pp. 822-825.

[4] I. Hajnsek, E. Pottier, and S.R. Cloude, "Inversion of surface parameters from polarimetric sar," Geoscience and Remote Sensing, IEEE Transactions on, vol. 41, no. 4, pp. 727-744, April 2003. 